\title{
Cooperative Beamforming and Modern Spatial Diversity Techniques for Power Efficient Wireless Sensor Networks
}

\author{
Tommy Hult \\ Lund University \\ Sweden \\ Abbas Mohammed and Zhe Yang \\ Blekinge Institute of Technology \\ Sweden
}

\section{Introduction}

Wireless Sensor Networks (WSN) have been attracting great attention recently. They are relatively low cost to be deployed and to be used in many promising applications, such as biomedical sensor monitoring (e.g., cardiac patient monitoring), habitat monitoring (e.g., animal tracking), weather monitoring (temperature, humidity, etc.), low-performance seismic sensing, environment preservation and natural disaster detection and monitoring (e.g., flooding and fire) Lewis (2004); Tubaishat \& Madria (2003); Stankovic et al. (2003); Akyildiz (2002); Rashid-Farrokhi et al. (1998).

The WSN applications analyzed in this chapter have a topology where a large number of wireless sensor nodes are spread out over a large or small geographic area (e.g., disaster regions, indoor factory, large sports event areas, etc.). In this topology, an inefficient use of bandwidth and transmitter power resources is resulted if each wireless sensor is transmitting its measurement data to the base station (processing central). In this case, each sensor node would have to be assigned its own frequency channel and, if the base station is located a long distance from the sensor nodes, it would also demand a higher than average sensor node transmitter power. By using a coordinating cluster head, for each cluster of wireless sensor nodes, we can instead use the combined transmitter power of the node cluster through the use of beamforming to increase the transmitter-receiver separation and/or to improve the signal-to-noise ratio (SNR) of the communication link. Another advantage of using this cooperative transmission is that we can exert power control to minimize the power consumption of each individual sensor node, and thus maximizing network lifetime. In addition, in a cooperative network the measurement data could be sent by using Time Division Multiplexing (TDM) instead of Frequency Division Multiplexing (FDM) which improves the overall bandwidth efficiency of the system.

The spatial properties of wireless communication channels are extremely important in determining the performance of the systems. Thus, there has been great interest in the application of beamforming and modern spatial diversity techniques (or multiantenna systems) since they 
can offer a broad range of ways to improve wireless systems performance. For instance, diversity techniques such as multiple-input single-output (MISO), single-input multiple-output (SIMO) and multiple-input multiple-output (MIMO) can enhance the capacity, coverage, quality and energy efficiency of of wireless systems.

Energy efficiency is one of the key requirements in many WSN applications. This is particularly crucial for WSN deployed in inaccessible or disaster environments in which battery recharging and replacement is not a viable option. Thus, in this chapter we first propose to use a cooperative beamforming approach in wireless sensor networks to increase the transmission range, minimize power consumption and maximize network lifetime. This will be of particular interest for outdoor applications, especially when monitoring remote areas using aerial vehicle, such as a High Altitude Platform (HAP) or Unmanned Aerial Vehicle (UAV), as a platform for the data collecting base station. We will investigate how the required transmitter power of each sensor node is affected by the number of cooperating transmission nodes in the network. In addition, we present a comparison in the use of beamforming with the different forms of modern spatial diversity techniques for the same purpose of achieving a longer transmission distance (or range) while maintaining a low energy consumption. Beamforming can of course be interpreted as a form of MISO system although it differs from the normal view of how a diversity system operates.

This chapter is organized as follows: Section 2 presents an overview and analysis of cooperative beamforming using a large aperture random array. In section 3, the MISO, SIMO and MIMO diversity schemes are introduced and analysed using the Rician fading channel employed in the simulations. Section 4 present numerical results and comparisons of the simulated beamformer and modern diversity systems. Finally, section 5 concludes the chapter.

\section{Traditional Cooperative Beamforming}

In this chapter we use the delay-and-sum beamforming technique which is the oldest and simplest algorithm for Space-Time processing. This beamforming is done through coherent excitation/reception of amplitude and phase of the signal transmitted/received from each individual antenna element in a collection or cluster of similar antenna elements also known as an antenna array Johnson (1993). Antenna arrays can have different configurations (e.g., linear, planar, circular, triangular, rectangular or spherical). Extensive research has been done on uniform array beamforming using one (linear) or two (planar) dimensional equi-distant element arrays Johnson (1993); Hansen \& Woodyard (1938); Drane (1968). In addition, there is also work done on beamforming using circular, triangular and rectangular arrays Johnson (1993); Balanis (1997).

The antenna array formed by individual sensor node antennas is assumed to be a planar array, of randomly positioned sensor node antennas, which is parallel with the plane containing all sensor nodes so that the sensor nodes are only extended in $x$ and $y$ direction and not in $z$ direction. This is a valid assumption in most cases since the elongation of the networks in $z$ direction in most cases is very small compared to the distance between the network cluster and the base station we want to communicate with Jenkins (1973). The design of this type of cooperative array is similar to the design of large aperture arrays where we have an interelement spacing that is random and larger than half the wavelength. There are no known simplifying techniques for synthesis of randomly spaced arrays, like Schelkunoffs polynomial method Johnson (1993); Balanis (1997) or the Fourier Transform method Johnson (1993); Balanis (1997). In the random array all properties, e.g., array pattern, beamwidth, sidelobe level and gain are stochastic variables. 
In figure 1, we show a scenario with $N=50$ sensor nodes deployed inside a circular boundary in the $x-y$ plane with a radius $R$. The sensor nodes are independent and uniformly distributed within the cluster area. The $n^{\text {th }}$ sensor then has the polar coordinates $\left(r_{n}, \phi_{n}\right)$.

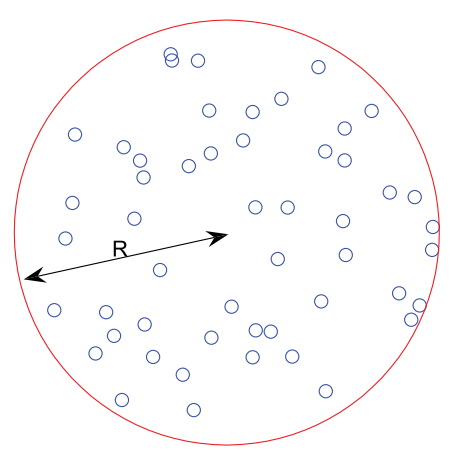

Fig. 1. The positioning of the employed sensor nodes within a cluster area of radius $R$ according to an independent uniform distribution.

The signal $y_{n}(t)$ at the array sensor node $n$ can then be expressed as,

$$
y_{n}(t)=s\left(t-\boldsymbol{\alpha}_{\mathbf{0}} \cdot \mathbf{x}_{\mathbf{0}}\right),
$$

where $s(t)$ is the signal to be transmitted/received and the $n^{\text {th }}$ sensor at location $x_{n}$ transmits/receives the electromagnetic signal $y_{n}(t)$. The slowness vector $\boldsymbol{\alpha}_{\mathbf{0}}$ is the required delay for each sensor to steer the array in a specific direction toward the signal source or target, and is defined as,

$$
\alpha_{0}=\frac{\mathrm{d}_{0}}{c}
$$

where $\mathbf{d}_{\mathbf{0}}$ is the direction of the wave propagation and $c$ is the speed of light. The total output of the delay-and-sum algorithm can be expressed by,

$$
z(t)=\sum_{n=0}^{N-1} w_{n} s\left(t+\left(\boldsymbol{\alpha}-\boldsymbol{\alpha}_{\mathbf{0}}\right) \cdot \mathbf{x}_{\mathbf{0}}\right),
$$

where $w_{n}$ is the amplitude weights of the array tapering and $\boldsymbol{\alpha}$ is the slowness vector for the direction of observation. If we assume that all the sensor nodes are approximately located in the same plane (i.e., the $x$-y plane) and the source/target is located at the spherical coordinates $\mathbf{d}_{\mathbf{0}}=\left(d_{0}, \phi_{0}, \theta_{0}\right)$ in the far-field, and we are transmitting a narrow band signal then we can approximate equation (3) as, (see appendix)

$$
G(\phi, \theta)=\frac{1}{N} \sum_{n=0}^{N-1} w_{n} e^{j \omega\left(t-\frac{r_{n}}{c}\left(\cos \left(\phi_{n}\right) u+\sin \left(\phi_{n}\right) v\right)\right.},
$$

where $u=\sin (\theta) \cos (\phi)-\sin \left(\theta_{0}\right) \cos \left(\phi_{0}\right)$ and $v=\sin (\theta) \sin (\phi)-\sin \left(\theta_{0}\right) \sin \left(\phi_{0}\right)$ for the direction of the incoming/outgoing wave $\left(\phi_{0}, \theta_{0}\right)$ and the direction of observation $(\phi, \theta)$. The function $G(\phi, \theta)$ is then one ensemble of the array amplitude gain function for one set of stochastic 
sensor locations. To find the ensemble mean of the array amplitude gain functions, we assume an independent uniform distribution of the sensor locations within the radius $R$,

$$
\mathrm{E}\{G(\phi, \theta)\}=\iint G(\phi, \theta) p_{R, \phi}\left(r_{n}, \phi_{n}\right),
$$

where $p_{R, \phi}\left(r_{n}, \phi_{n}\right)$ is the probability density function (PDF) of the sensor locations.

In figure 2 we show the absolute squared average array gain function $|E\{G(\phi, \theta)\}|^{2}$ of 250 realizations of the array amplitude gain function $G(\phi, \theta)$, and in figure 3 we show the standard deviation for the distribution of the amplitude sidelobe levels. From figure 2 we can also estimate a mean sidelobe level that will converge toward $\approx-17 \mathrm{~dB}$ which is consistent with the theoretical value, $N^{-1}$. The average signal-to-noise ratio of the array is defined as $S N R_{\text {array }}=$ $S N R_{\text {node }} \cdot G(\phi, \theta)$ which means that the array average SNR is $S N R_{\text {array }}=N \cdot S N R_{\text {node }}$ when we are aiming the array toward the incoming assumed plane wave. The $S N R_{\text {array }}$ is a Gaussian distributed parameter with a mean of $17 \mathrm{~dB}$, and a $95 \%$ confidence that the SNR of the array will be higher than $7 \mathrm{~dB}$.

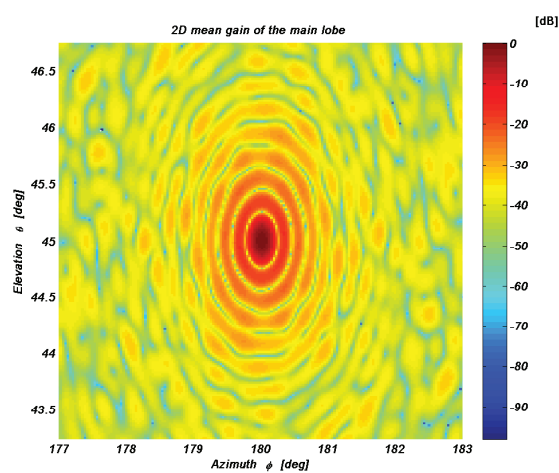

Fig. 2. The absolute squared average array pattern of 250 realizations of the random sensor locations. Only a small part around the main lobe is shown in the figure.

\section{Modern Spatial Diversity Techniques}

Another recently popular technique to improve the signal to noise ratio of the long range transmission is to use some form of spatial multiantenna diversity system. In this chapter, we employ modern diversity techniques which have gained great interest in the past decade or so. These are: multiple-input single-output (MISO), single-input multiple-output (SIMO) and multiple-input multiple-output (MIMO) antenna systems. Multiple transmit and receive antenna systems allow increased data rates and enhanced link reliability of wireless communication systems while reducing the transmission power requirements. In the following analysis of these diversity techniques, we will assume a perfect knowledge of the propagation channel.

\subsection{Cooperative Multiple-Input Single-Output}

Consider a frequency flat fading propagation model with $N_{t x}$ antenna elements at the transmitter and one antenna element at the receiver. To take full advantage of the antenna transmit 


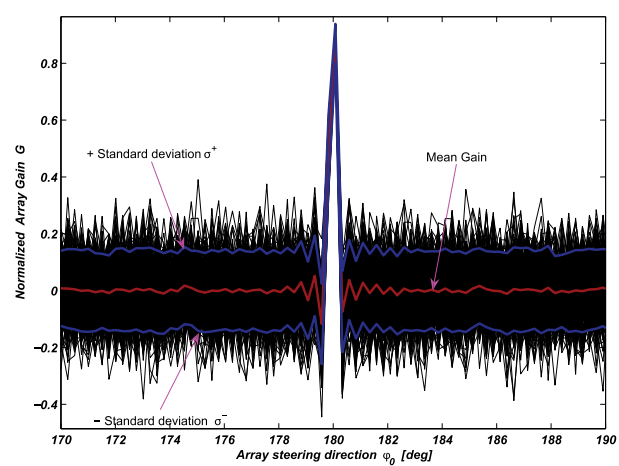

Fig. 3. A plot showing a cross-section of the main lobe of all 250 realizations of the array amplitude gain pattern.

diversity we send multiple weighed copies of the signal sample through all the transmitting antenna elements. The received baseband signal sample can then be expressed as,

$$
r[m]=\sqrt{\frac{E_{s}}{N_{t x}}} \sum_{l=0}^{L-1} h_{l} w_{l} s[m]+n[m],
$$

where $r[m] \in \mathbb{C}$ is the received sample, $s[m] \in \mathbb{C}$ is the transmitted sample and $n[m]$ is a noise sample with $n[m] \sim \mathcal{C N}\left(0, \sigma_{n}^{2}\right)$. The coefficient $w_{l}$ is the channel weight for channel $l$ and $E_{s}$ is the transmitted average symbol energy. This can be expressed in vector notation as,

$$
r=\sqrt{\frac{E_{s}}{N_{t x}}} \mathbf{h w s}+n,
$$

where $\mathbf{h} \in \mathbb{C}^{N_{t x} \times 1}$ is the frequency of flat fading channel vector with a Rice distribution. The normalized Rician channel vector $\mathbf{h}$ can then be defined as, (McKay et al., 2006)

$$
\mathbf{h} \triangleq \sqrt{c_{1}} \mathbf{l}+\sqrt{c_{2}} \mathbf{R}_{t x} \mathbf{h}_{n}
$$

where $\mathbf{1}$ is the line-of-sight (LOS) component represented as a mean value that satisfies the condition $|\mathbf{1}|^{2}=N_{t x}$, and $\mathbf{R}_{t x}$ is the transmit correlation vector. $\mathbf{R}_{t x}$ is assumed to be positive definite full rank matrix. $\mathbf{h}_{n} \sim \mathcal{C N}_{N_{t x}}\left(\mathbf{0}_{N_{t x}}, \mathbf{1}_{N_{t x}}\right)$ is a complex valued Gaussian vector representing the non line-of-sight (NLOS) component. The coefficients $c_{1}=K /(K+1)$ and $c_{2}=1 /(K+1)$ are normalizing factors, where $K$ is the Rice factor which represents the power ratio between the LOS and NLOS components. The weight vector $\mathbf{w}$ that maximizes the received SNR is given by,

$$
\mathbf{w}=\sqrt{N_{t x}} \frac{\mathbf{h}^{H}}{\|\mathbf{h}\|},
$$

which is the transmit maximum ratio combining (MRC) method and is also known as matched beamforming. The SNR of the received signal can then be expressed as,

$$
\gamma_{r x}=\frac{E_{S} \cdot|\mathbf{h}|^{2}}{N_{0}} .
$$




\subsection{Cooperative Single-Input Multiple-Output}

The second type of spatial diversity is receive diversity in which we are utilizing a single-input multiple-output (SIMO) frequency flat fading propagation channel model with $N_{r x}$ receiving antenna elements and a single transmitting antenna element. To fully exploit the receive diversity we will receive multiple copies of the transmitted signal through all the $N_{r x}$ receiving antenna elements. The received baseband signal sample can then be expressed as,

$$
r[m]=\sqrt{\frac{E_{s}}{N_{r x}}} \sum_{l=1}^{L}\left(w_{l} h_{l}\right) s[m]+\sum_{l=1}^{L} w_{l} n_{l}[m],
$$

where $r_{l}[m] \in \mathbb{C}$ is the received sample from receiving antenna element $l, s[m] \in \mathbb{C}$ is the transmitted sample and $n_{l}[m]$ is a noise sample at receiving antenna element $l$ with $n_{l}[m] \sim$ $\mathcal{C N}\left(0, \sigma_{n}^{2}\right)$. the coefficient $w_{l}$ is the channel weight at receiving antenna element $l$ and $E_{s}$ is the transmitted average symbol energy. This can be expressed in vector notation as,

$$
r=\sqrt{E_{s}} \mathbf{w}^{H} \mathbf{h} s+\mathbf{w}^{H} \mathbf{n},
$$

where $\mathbf{h} \in \mathbb{C}^{N_{t x} \times 1}$ is the frequency flat fading channel vector with a Rice distribution. The normalized channel vector $\mathbf{h}$ can then be defined as, (McKay et al., 2006)

$$
\mathbf{h} \triangleq \sqrt{c_{1}} \mathbf{1}+\sqrt{c_{2}} \mathbf{R}_{r x} \mathbf{h}_{n}
$$

where $\mathbf{l}$ is the line of sight (LOS) component represented as a mean value that satisfies the condition $|\mathbf{1}|^{2}=N_{r x}$, and $\mathbf{R}_{r x}$ is the receive correlation vector. $\mathbf{R}_{r x}$ is assumed to be a positive definite full rank matrix. $\mathbf{h}_{n} \sim \mathcal{C} \mathcal{N}_{N_{r x}}\left(\mathbf{0}_{N_{r x}}, \mathbf{1}_{N_{r x}}\right)$ is a complex valued Gaussian vector representing the nnon-line-of-sight (NLOS) component. The weight vector $\mathbf{w}$ that maximize the received SNR at each antenna element is given by,

$$
\mathbf{w}=\sqrt{N_{r x}} \frac{\mathbf{h}^{H}}{\|\mathbf{h}\|} .
$$

The SNR of the received signal after we have performed a maximum ratio combining (MRC) can then be expressed as

$$
\gamma_{r x}=\frac{E_{s} \cdot|\mathbf{h}|^{2}}{N_{0}}
$$

\subsection{Cooperative Multiple-Input Multiple-Output}

By combining the MISO and SIMO diversity techniques we create a system of $\left(N_{t x}\right.$ and $\left.N_{r x}\right)$ transmitting and receiving antenna elements, respectively, which is known as a multiple-input multiple-output (MIMO) system. If we consider a frequency flat fading $\left(N_{t x} \times N_{r x}\right)$ MIMO propagation model, the received signal can be written in vector notation as,

$$
r=\sqrt{\frac{E_{s}}{N_{t x}}} \mathbf{w}_{r x}^{H} \mathbf{H} \mathbf{w}_{t x} S+\mathbf{w}_{r x} \mathbf{n} .
$$

In the MIMO case, the Rice distributed channel matrix $\mathbf{H}$ can be derived as,

$$
\mathbf{H} \triangleq \sqrt{c_{1}} \mathbf{L}+\sqrt{c_{2}} \mathbf{R}_{r x}^{\frac{1}{2}} \mathbf{H}_{n} \mathbf{R}_{t x}^{\frac{1}{2}}
$$


where $\mathbf{L}$ represents the LOS component and is the arbitrary rank mean value matrix with the condition that $\operatorname{Tr}\left(\mathbf{L} \mathbf{L}^{H}\right)=N_{r x} \cdot N_{t x}, \mathbf{R}_{r x}$ and $\mathbf{R}_{t x}$ are the correlation matrices on the transmitter and receiver side respectively. $\mathbf{H}_{n} \sim \mathcal{C} \mathcal{N}_{N_{r x}, N_{t x}}\left(\mathbf{0}_{N_{r x} \times N_{t x}}, \mathbf{I}_{N_{r x}} \otimes \mathbf{I}_{N_{r x}}\right)$.

To maximize the combined SNR at the receiver antenna elements we maximize,

$$
\gamma_{r x}=\frac{E_{s}}{N_{0}} \cdot \frac{\left\|\mathbf{w}_{r x}^{H} \mathbf{H} \mathbf{w}_{t x}\right\|^{2}}{N_{t x}\left\|\mathbf{w}_{r x}\right\|^{2}} .
$$

$\gamma_{r x}$ is then maximized when $\mathbf{w}_{r x}$ and $\mathbf{w}_{t x} / N_{t x}$ are equal to the singular input and output vectors of the channel matrix $\mathbf{H}$ corresponding to the maximum singular value of the channel matrix $\mathbf{H}$. Equation 16 can then be written as,

$$
r[m]=\sqrt{E_{s}} \sigma_{\max } s[m]+n[m] .
$$

where $\sigma_{\max }$ is the maximum singular value of the channel matrix $\mathbf{H}$ and since $\sigma_{\max }^{2}$ is the same as the maximum eigenvalue $\lambda_{\max }$ of $\mathbf{H H}^{H}$. We can now express the received SNR of the MIMO diversity technique as,

$$
\gamma_{r x}=\frac{E_{s}}{N_{0}} \cdot \lambda_{\max }
$$

\section{Simulation Results}

In this section we assess the performance of beamforming technique and modern spatial diversity techniques and compare the results with the nondiversity single antenna (or SISO) system. If we consider a base station mounted on an aerial platform such as a HAP or a UAV to collect data from remote sensor networks, then the amount of obstructions in the transmission path would depend on the type of environment at the sensor locations, although it can still generally be assumed that the number of obstructions will increase with a decreasing antenna elevation angle. Therefore, the propagation effect of the change in elevation can be translated into a change of the Rice distribution K-factor.

In the presented simulations, the Rician K-factor was varied over an interval of $K \in$ $\left[1 \cdot 10^{-8}, 1 \cdot 10^{+8}\right]$, where the low value represents a channel with no LOS component and very little correlation between the different signal paths and therefore resembles a Rayleigh fading channel. When the Rician K-factor is gradually increased the correlation between the signal paths will increase and the Direction of Departure (DoD)/Direction of Arrival (DoA) of the signals will narrow into a smaller and smaller angular sector, until the K-factor asymptotically goes toward infinity and all signal paths will be correlated and pointing in the same direction.

In figure 4 we see the comparison between the ordinary random array beamformer performance and the MISO/SIMO diversity systems performance. Inspecting figure 4, we can see that the MISO/SIMO diversity system seems to maintain a constant low node transmitter power $P_{t x}$ even in a NLOS scenario by spreading the energy over multiple paths instead of transmitting it all in one direction. Furthermore, we can see from figure 4 that if the distance between the transmitting nodes and the basestation is increased from $1 \mathrm{~km}$ to $10 \mathrm{~km}$, the nodes need a 100 fold increase of the total transmitted power to maintain the same capacity. This is independent of whether we are using the nodes as a beamforming array or a diversity system, which is consistent with the inverse square law of the free space loss.

Finally, we assess the performance of the full multiantenna diversity system (or MIMO) where we have multiple antenna nodes on both the transmitting and receiving end of the link. In 
addition, we compare the results with the conventional array beamformer, with its subsets (SIMO/MISO) and the nondiversity single antenna (or SISO) system. In the results shown in figure 5 we increase the number of receiving antenna nodes to be equal to the number of transmitting antenna nodes to get a $(50 \times 50)$ MIMO system which will increase the array and diversity gains even further. This effect can clearly be seen in figure 5 where the performance of the MIMO system outperforms the other systems in both LOS and NLOS scenarios. It is also clear from this figure that the nondiversity SISO system and the conventional beamformer will not function properly in this setting and in particular in NLOS conditions. These initial results suggest that the application of modern spatial diversity systems is expected to improve the energy efficiency, lifetime and the overall performance of the wireless sensor network.

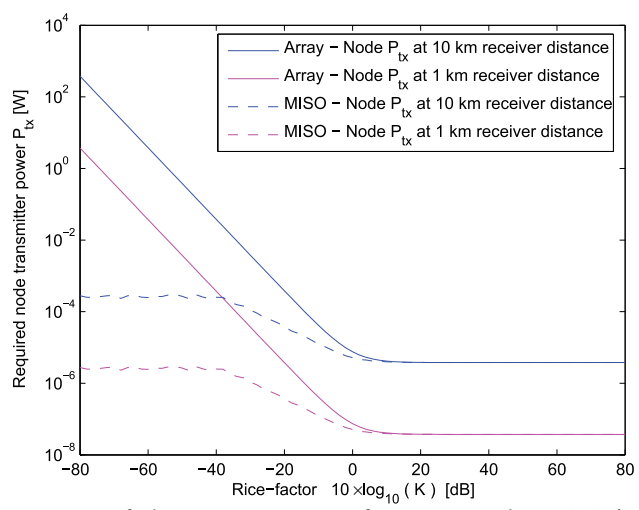

Fig. 4. Comparison between of the Array Beamformer and MISO/SIMO system for different $\mathrm{K}$-factor values for a distance from the base station of $1 \mathrm{~km}$ and $10 \mathrm{~km}$, respectively.

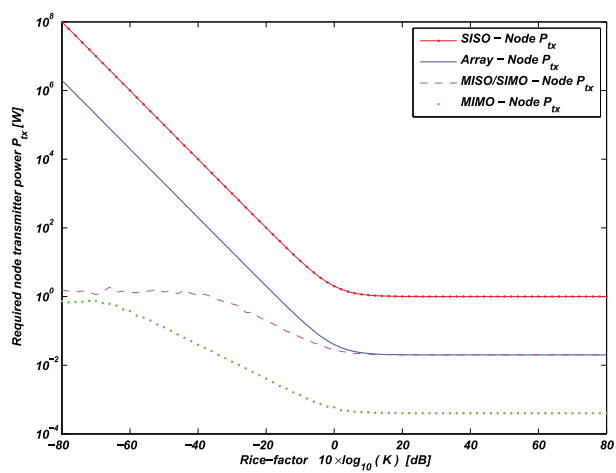

Fig. 5. Performance of the Array Beamformer, MISO/SIMO and MIMO systems for different $\mathrm{K}$-factor values and compared with a single antenna SISO system. The performance results are normalised against SISO in this figure. 


\section{Conclusions}

In this chapter we have investigated how the required transmitter power of each sensor node is affected by the number of cooperating transmission nodes in a traditional random beamformer array. Due to the randomness of the sensor node positions, there is no simple algorithm for mitigation of interference from a fixed direction. This is because the sidelobe levels and the sidelobe positions are random. A comparison in the use of beamforming with modern diversity systems such as MISO/SIMO and MIMO for the same purpose of achieving a longer transmission distance or maintaining a low energy consumption is also presented. It is clear from these investigations that the MISO/SIMO and MIMO diversity systems are superior in performance to both the SISO link and the traditional form of array beamforming, especially when the LOS component is small or non-existent. Even one extra antenna at the receiving base station will increase the performance of the system two-fold in a LOS scenario and give an improved performance in NLOS as well. The best performance though, is given by the MIMO system where we have multiple antenna nodes on both the transmitting and receiving end of the link. Initial results suggest that the application of modern spatial diversity systems is expected to improve the energy efficiency and lifetime of wireless sensor network.

\section{Appendix: Derivation of Equation (3)}

The slowness vector $\boldsymbol{\alpha}$ in (2) is defined as,

$$
\boldsymbol{\alpha}=\frac{\mathbf{d}}{c} .
$$

The $\mathbf{d}$ vector represents the direction of observation and can be expressed in cartesian coordinates as,

$$
\mathbf{d}=d \cdot\{-\sin (\theta) \cos (\varphi),-\sin (\theta) \sin (\varphi), \cos (\theta)\} .
$$

Assuming that the sensor nodes are only distributed in the $x-y$ plane. In addition, if we assume a far-field plane wave solution, then the individual propagation induced time delay $\Delta t_{n}$ is calculated from the slowness vector $\boldsymbol{\alpha}$ and the position vector $\mathbf{x}_{n}$ of each node $n$ as,

$$
\begin{gathered}
\Delta t_{n}=\boldsymbol{\alpha} \cdot \mathbf{x}_{n}=\frac{r_{n}}{c}\left(-\sin (\theta) \cos (\varphi) \cos \left(\varphi_{n}\right)-\sin (\theta) \sin (\varphi) \sin \left(\varphi_{n}\right)-0\right) \\
\Delta t_{n}=-\frac{r_{n}}{c}\left(\sin (\theta) \cos \left(\varphi-\varphi_{n}\right)\right)
\end{gathered}
$$

The actual direction of propagation $\mathbf{d}_{0}$ is used to calculate the slowness vector $\boldsymbol{\alpha}_{0}$ of the centre point of the array,

$$
\Delta t_{0}=\frac{r_{n}}{c}\left(\sin (\theta) \cos \left(\varphi_{0}-\varphi_{n}\right)\right)
$$

Substituting (24) and (25) into (3) results in,

$$
z(t)=\sum_{n=0}^{N-1} w_{n} s\left(t-\frac{r_{n}}{c}\left(\left(\sin (\theta) \cos \left(\varphi-\varphi_{n}\right)\right)-\left(\sin (\theta) \cos \left(\varphi_{0}-\varphi_{n}\right)\right)\right)\right) .
$$

Denoting $u=\sin (\theta) \cos (\phi)-\sin \left(\theta_{0}\right) \cos \left(\phi_{0}\right), v=\sin (\theta) \sin (\phi)-\sin \left(\theta_{0}\right) \sin \left(\phi_{0}\right)$ and assuming a sinusoidal signal $s(t),(26)$ can be expressed as a time harmonic solution,

$$
G(\phi, \theta)=\frac{1}{N} \sum_{n=0}^{N-1} w_{n} e^{j \omega\left(t-\frac{r_{n}}{c}\left(\cos \left(\phi_{n}\right) u+\sin \left(\phi_{n}\right) v\right)\right.} .
$$




\section{References}

Akyildiz, I.; Su, W.; Sankarasubramaniam, Y. \& Cayirci, E. (2002). A survey on wireless sensor networks. IEEE Communications Magazine, Vol. 40, No. 8, August 2002, 102-114.

Balanis, C. (1997). Antenna Theory: Analysis and Design, Chapter 6, John Wiley, 1997.

Cui, S.; Goldsmith, A. \& Bahai, A. (2004). Energy-efficiency of MIMO and cooperative MIMO techniques in sensor networks. IEEE Journal on Selected Areas in Communications, Vol. 22, No. 6, August 2004, 1089-1098.

Drane, C. Jr. (1968). Useful approximations for the directivity and beamwidth of large scanning Dolph-Chebyshev arrays. IEEE Proceedings, Vol. 56, No. 11, November 1968, 1779-1787.

Hansen, W. \& Woodyard, R. (1938). A New Principle in Directional Antenna Design, Proceedings IRE, Vol. 26, No. 3, March 1938, 333-345.

Jenkins, J. (1973). Some properties and examples of random listening arrays. IEEE Oceans, Vol. 5, September 1973, 466-469.

Johnson, D. \& Dudgeon, D. (1993). Array Signal Processing: concepts and techniques, PrenticeHall Inc., 1993, ISBN 0-13-048513-6.

Lewis, F. (2004). Wireless sensor networks, In: Smart Environments: Technologies, Protocols, and Applications, Cook, D. \& Das, S. (Editors), John Wiley, New York, 2004.

McKay, M. \& Collings, I. (2006). Improved general lower bound for spatially-correlated Rician MIMO capacity. IEEE Communications Letters, Vol. 10, No. 3, March 2006, 162-164.

Pillutla, L. \& Krishnamurhty, V. (2005). Joint rate and cluster optimization in cooperative MIMO sensor networks. IEEE 6th Workshop on Signal Processing Advances in Wireless Communications, June 2005, 265-269.

Rashid-Farrokhi, F.; Tassiulas L. \& and Liu, K. (1998). Joint power control and beamforming in wireless networks using antenna arrays. IEEE Transactions on Communication, Vol. 46, No. 10, October 1998, 1313-1324.

Stankovic, J.; Abdelzaher, T.; Lu, C.; Sha, L. \& Hou, J. (2003). Realtime communication and coordination in embedded sensor networks. Proceedings of The IEEE, Vol. 91, No. 7, July 2003, 1002-1022.

Tubaishat, M. \& Madria, S. (2003). Sensor networks: an overview. IEEE Potentials, Vol. 22, No. 2, April 2003, 20-23. 


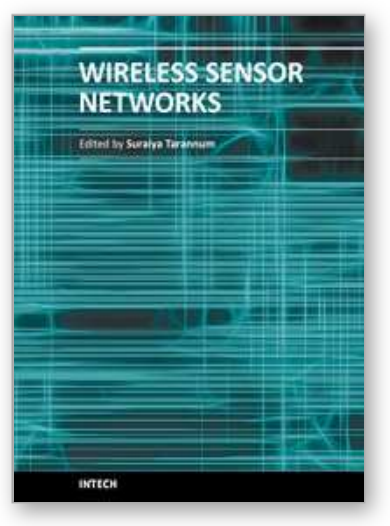

\section{Wireless Sensor Networks \\ Edited by}

ISBN 978-953-307-325-5

Hard cover, 342 pages

Publisher InTech

Published online 29, June, 2011

Published in print edition June, 2011

\section{How to reference}

In order to correctly reference this scholarly work, feel free to copy and paste the following:

Tommy Hult, Abbas Mohammed and Zhe Yang (2011). Cooperative Beamforming and Modern Spatial Diversity Techniques for Power Efficient Wireless Sensor Networks, Wireless Sensor Networks, (Ed.), ISBN: 978-953-307-325-5, InTech, Available from: http://www.intechopen.com/books/wireless-sensornetworks/cooperative-beamforming-and-modern-spatial-diversity-techniques-for-power-efficient-wirelesssensor-

\section{INTECH}

open science | open minds

\author{
InTech Europe \\ University Campus STeP Ri \\ Slavka Krautzeka 83/A \\ 51000 Rijeka, Croatia \\ Phone: +385 (51) 770447 \\ Fax: +385 (51) 686166 \\ www.intechopen.com
}

\author{
InTech China \\ Unit 405, Office Block, Hotel Equatorial Shanghai \\ No.65, Yan An Road (West), Shanghai, 200040, China \\ 中国上海市延安西路65号上海国际贵都大饭店办公楼 405 单元 \\ Phone: +86-21-62489820 \\ Fax: $+86-21-62489821$
}


(C) 2011 The Author(s). Licensee IntechOpen. This chapter is distributed under the terms of the Creative Commons Attribution-NonCommercialShareAlike-3.0 License, which permits use, distribution and reproduction for non-commercial purposes, provided the original is properly cited and derivative works building on this content are distributed under the same license. 